\title{
The C. elegans Hox gene ceh-13 regulates cell migration and fusion in a non-colinear way. Implications for the early evolution of Hox clusters
}

\author{
Borbála Tihanyi ${ }^{1}$, Tibor Vellai ${ }^{1 *}$, Ágnes Regős ${ }^{1}$, Eszter Ari ${ }^{1}$, Fritz Müller ${ }^{2}$, Krisztina Takács-Vellai ${ }^{1}$
}

\begin{abstract}
Background: Hox genes play a central role in axial patterning during animal development. They are clustered in the genome and specify cell fate in sequential domains along the anteroposterior (A-P) body axis in a conserved order that is co-linear with their relative genomic position. In the soil worm Caenorhabditis elegans, this striking rule of co-linearity is broken by the anterior Hox gene ceh-13, which is located between the two middle Hox paralogs, lin-39 and mab-5, within the loosely organized nematode Hox cluster. Despite its evolutionary and developmental significance, the functional consequence of this unusual genomic organization remains unresolved.
\end{abstract}

Results: In this study we have investigated the role of ceh-13 in different developmental processes, and found that its expression and function are not restricted to the anterior body part. We show that ceh-13 affects cell migration and fusion as well as tissue patterning in the middle and posterior body regions too. These data reveal novel roles for ceh-13 in developmental processes known to be under the control of middle Hox paralogs. Consistently, enhanced activity of lin-39 and mab-5 can suppress developmental arrest and morphologic malformation in ceh-13 deficient animals.

Conclusion: Our findings presented here show that, unlike other Hox genes in C. elegans which display regionspecific accumulation and function along the A-P axis, the expression and functional domain of the anterior Hox paralog ceh-13 extends beyond the anterior region of the worm. Furthermore, ceh-13 and the middle Hox paralogs share several developmental functions. Together, these results suggest the emergence of the middle-group Hox genes from a ceh-13-like primordial Hox ancestor.

\section{Background}

One of the most striking shared developmental mechanisms in divergent animal phyla is the patterning of the anteroposterior body axis by evolutionarily conserved homeodomain-containing transcription factors encoded by Hox genes [1-5]. Properties of the Hox genes include clustering in the genome, a conserved order within the cluster, a co-linear arrangement of their genomic position and functional domain in the body, and a hierarchy of action between the adjacent Hox paralogs. In C. elegans, the Hox cluster consists of six Hox genes that represent

\footnotetext{
* Correspondence: vellai@falco.elte.hu
'Department of Genetics, Eötvös Loránd University, Budapest, H-1117,

* Correspondence: vellai@falco.elte.hu Hungary
}

(c) 2010 Tihanyi et al; licensee BioMed Central Ltd. This is an Open Access article distributed under the terms of the Creative Commons Attribution License (http://creativecommons.org/licenses/by/2.0), which permits unrestricted use, distribution, and reproduction in any medium, provided the original work is properly cited.
(C. elegans homeobox-containing gene), two middlegroup paralogs, lin-39 (lineage defective) and mab-5 (male abnormal), and three posterior paralogs, egl-5 (egglaying defective), nob-1 (no backside) and php-3 (posterior Hox gene paralog) (Figure 1) [6-14].

The C. elegans Hox genes differ in some characteristics from their counterparts in other animal phyla. For example, embryonic patterning and viability in C. elegans require only the anterior and the two most posterior $H o x$ genes; triple loss-of-function (lf) mutant worms defective for lin-39, mab-5 and egl-5 can develop into fertile adults $[3,15]$, raising interesting questions about the evolutionary status of these dispensable Hox genes. They control various developmental processes, including the migration 


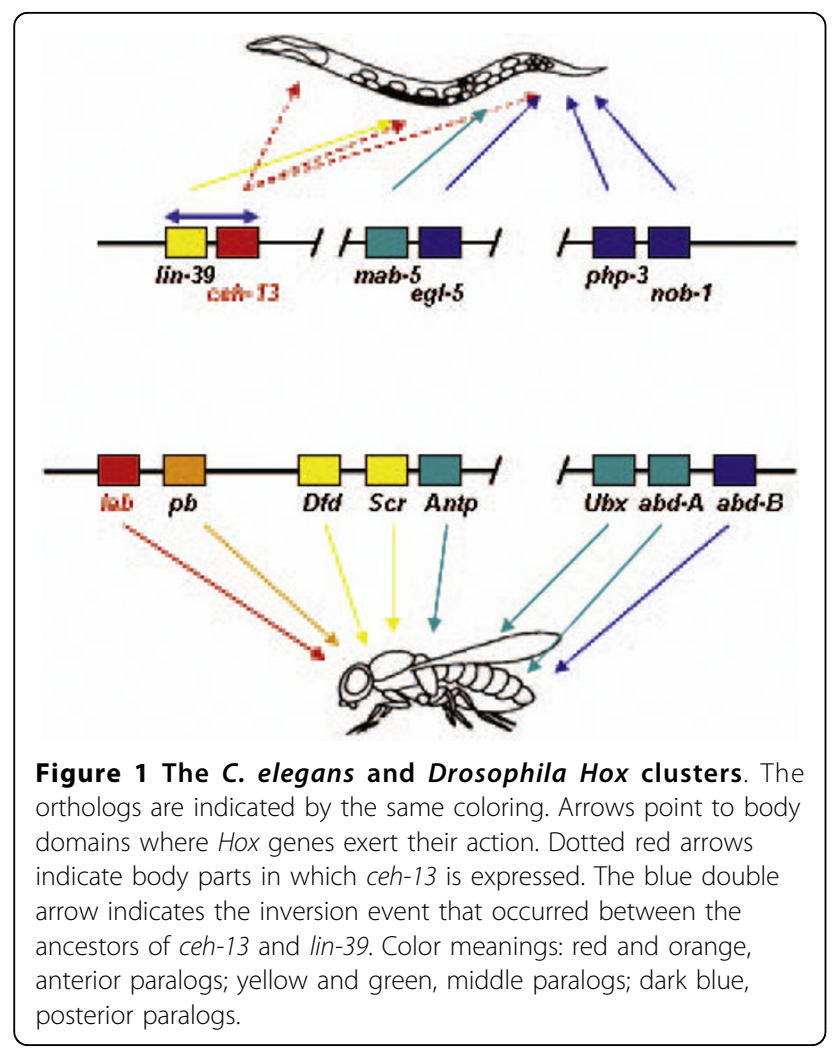

of Q neuroblasts, cell fusion in Pn.p cell lineages, cell fate specification during vulval patterning, and programmed cell death [11,12,16-18].

Another unique feature of the C. elegans Hox genes is the unusual position of the anterior Hox paralog ceh-13, the nematode counterpart of Drosophila labial and mammalian HoxB1 $[9,13,14]$. ceh-13 is located downstream of the middle Hox gene lin-39, the worm ortho$\log$ of Drosophila Deformed/Sex comb reduced and mammalian HoxD4 (Figure 1), thereby representing a break in co-linearity. The functional consequence of this unusual genomic organization and the role of ceh-13 in development remain largely unknown.

sw1, a strong lf mutation in ceh-13, disrupts normal patterning of the anterior body part and arrests development during embryonic or early larval stages [14]. However, a small percent of ceh-13(sw1) mutants are able to develop into fertile adults, suggesting that this gene shares developmental roles with (an)other Hox paralog(s). Regardless of the anterior manifestation of the pleiotropic Ceh-13 mutant phenotype, ceh-13 displays a complex, highly dynamic expression pattern, which involves several different cell lineages all over the body, even in the developing tail region $[13,19,20]$. These data raise the intriguing possibility that the influence of ceh-13 on cell fate specification may not be restricted to the anterior body part, and its functional domain may overlap with that of other Hox paralogs.

The proper function of HOX proteins requires TALE homeodomain proteins $[21,22]$. In C. elegans, these HOX cofactors are encoded by ceh-20 and unc-62 (uncoordinated), the orthologs of Extradenticle/Pbx and Homothorax/Meis/Prep genes, respectively [23-26]. Interestingly, the function of $\mathrm{CEH}-20$ and UNC-62 is partly independent of LIN-39 and MAB-5 in regulating cell migration and fusion as well as vulval cell fate specification in the mid- and posterior body regions $[23,25,26]$. This indicates a potential role for (an)other Hox gene(s) in the control of these developmental processes.

In this study we have implicated a role for ceh-13 in positioning $\mathrm{Q}$ neuroblasts and the fusion process of $\mathrm{Pn}$. p cells. Unexpectedly, the function of ceh-13 in these paradigms was obvious along the entire anteroposterior body axis. In addition, vulva patterning also appeared to be affected in ceh-13 mutant animals. Consistently, we found that the expression domain of ceh-13 overlaps with those of the other Hox paralogs, and ceh-13 interacts with lin-39 and mab-5 as the elevated levels of which are able to suppress the embryonic and early larval lethal phenotype of ceh-13 lf mutants. These findings suggest that in the nematode lineage the middle Hox genes emerged from a primordial ceh-13-like Hox paralog, and that the ancestor of this anterior Hox paralog might have given raise the primitive Hox cluster through tandem gene duplications during an early phase of animal evolution.

\section{Results}

ceh-13 is required for the positioning of $Q$ neuroblasts

Positioning of $\mathrm{Q}$ cell descendants provides an excellent paradigm to study how Hox genes affect cell migration during development. The QR and QL neuroblasts are born by the division of the $\mathrm{Q}$ cell in the posterior body part of the animal, and initially located directly opposite to each other. During the early larval stages, QR and then its descendants migrate toward the anterior, while QL and its descendants migrate posteriorly (Figure 2A) [27]lin-39 and mab-5 control the migration of these cells in a concerted manner $[11,12]$. The migration of QR and its descendants requires lin-39 activity: lin-39 deficiency blocks the migration of these cells prematurely at various positions. The migration of QL and its descendants is influenced by mab-5. Inactivation of mab-5 renders these cells to be incapable of migrating posteriorly; instead, they migrate toward the head. Conversely, a gain-of-function (gf) allele of mab-5, e1751, causes QR, which normally moves anteriorly, to migrate toward the tail (Figure 2B, C) $[11,12]$. The migration of Q neuroblasts is also influenced by ceh-20 and unc-62, 


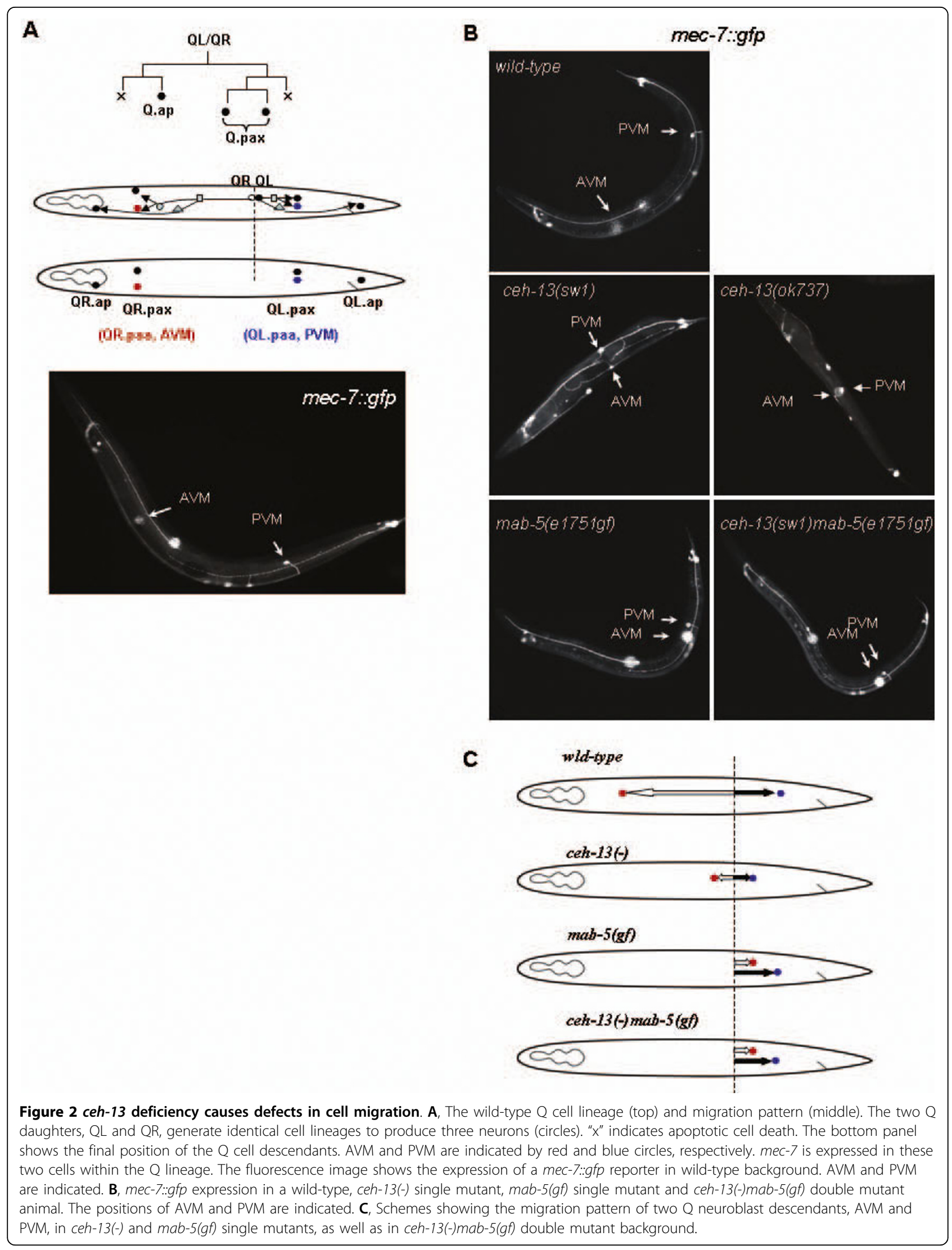


which are predicted to function as cofactors of lin-39 and $m a b-5$ in these processes. However, lin-39(-)ceh-20 $(-)$ and mab-5(-)ceh-20(-) double mutant animals exhibit more severe defects in the migration of these cells than either of these single Hox mutants [25]. This suggests a role for $\mathrm{CEH}-20$ in this cell migration paradigm which is independent of LIN-39 and MAB-5.

To examine whether CEH-13 influences cell migration in the $\mathrm{Q}$ cell lineage, we monitored the final position of two Q descendants, QR.paa (AVM) and QL.paa (PVM), in wild-type versus ceh-13 if mutant background. To visualize these cells, we used an integrated green fluorescent protein- (GFP) labeled mec-7 reporter [28], which is expressed only in these two neurons within the $\mathrm{Q}$ lineage (Figure 2A). We found that in ceh-13(sw1) mutant animals, AVM stops to migrate toward the anterior prematurely (Figs. 2B, C and 3). As a result, AVM

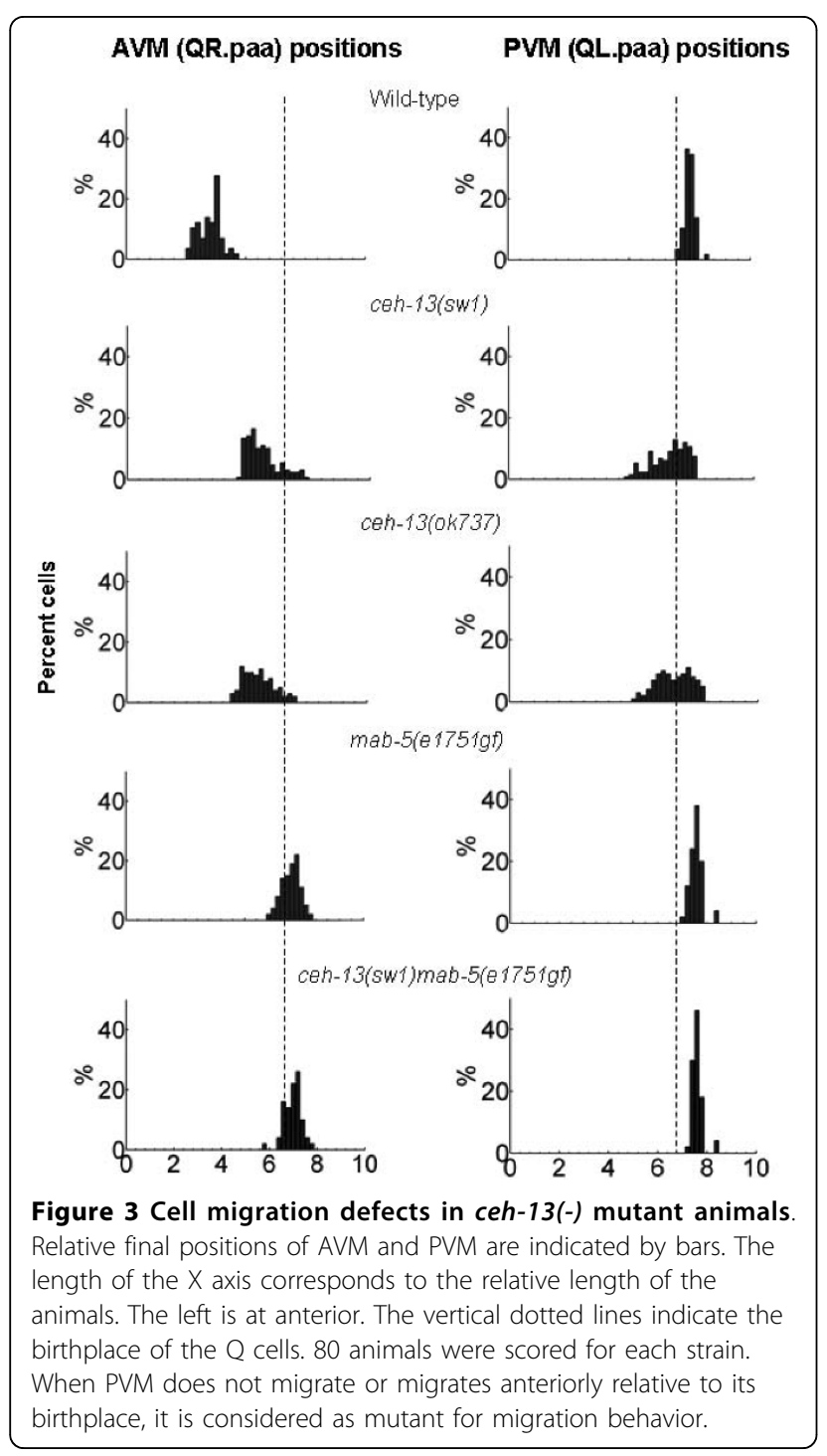

was often located close to the central body region. This mutant phenotype was expressed with almost a full penetrance in ceh-13(sw1) mutant larvae. A similar Q cell lineage-specific migration defect was observed in ceh-13(ok737) mutants too (Figure 2B). We next assayed cell migration in the QL lineage in ceh-13 deficient background. To our surprise, defects in QL lineage positioning were also evident in ceh-13(sw1) mutant animals: PVM was located improperly in $22 \%$ (19 out of 86 ) of the animals examined (Figs. 2B, C and 3). In the affected larvae, this cell was unable to migrate to its normal final position. Although this migration defect was partially penetrant, we conclude that the functional domain of ceh-13 in controlling Q cell migration overlaps with that of lin-39 and mab-5.

The mab-5(e1751gf) mutation reverses the direction of AVM migration, but does not affect PVM migration $[11,12]$. We also analyzed the position of Q cell descendants in ceh-13(sw1)mab-5(e1751gf) double mutant animals, and found that AVM is located slightly posterior to the position where $\mathrm{QL}$ and $\mathrm{QR}$ are normally born (Figs. 2B, C and 3). Thus, ceh-13 and mab-5 may have opposite effects on this particular cell migration event, and the combination of the two mutations may inhibit the ability of AVM and its progenitors to migrate to their normal position.

Normal positioning of AVM also requires mig-13 (cell migration abnormal), which codes for a novel transmembrane receptor [29]. The expression of mig-13 is restricted to the anterior body part by the inhibition of mab-5: mig-13 is normally active in certain cells of the ventral nerve cord (VNC) in the anterior half of the L1 stage larvae, but becomes ectopically expressed in the posterior body part in mab-5 If mutants [29]. Since ceh-13 is also expressed in the VNC at this stage [14], we asked whether ceh-13 interacts with mig-13 to control AVM migration. We found that AVM displays more severe positioning defects in ceh-13(sw1); mig-13(mu294) double mutant animals than in either of the single mutants (Figure 4A-C). In a portion of these double mutants, AVM was positioned even toward the posterior (Figure 4A-C). In good accordance with these results, mig-13::gfp expression was completely abolished in ceh-13 mutant larvae (data not shown). In some ceh-13 deficient larvae, even the pharyngeal-intestinal valve cells, which always show a strong mig13 expression throughout all larval stages in wild-type background, failed to express mig-13 (Figure 4D). These data raise the possibility that ceh-13 may control the anterior migration of cells in the Q lineage via influencing mig-13 activity. In summary, we conclude that ceh-13 is required for the normal positioning of both QR and QL descendants. The regulation of cell migration in the posterior part of the body by the anterior-like Hox gene ceh-13 was somehow unexpected. 


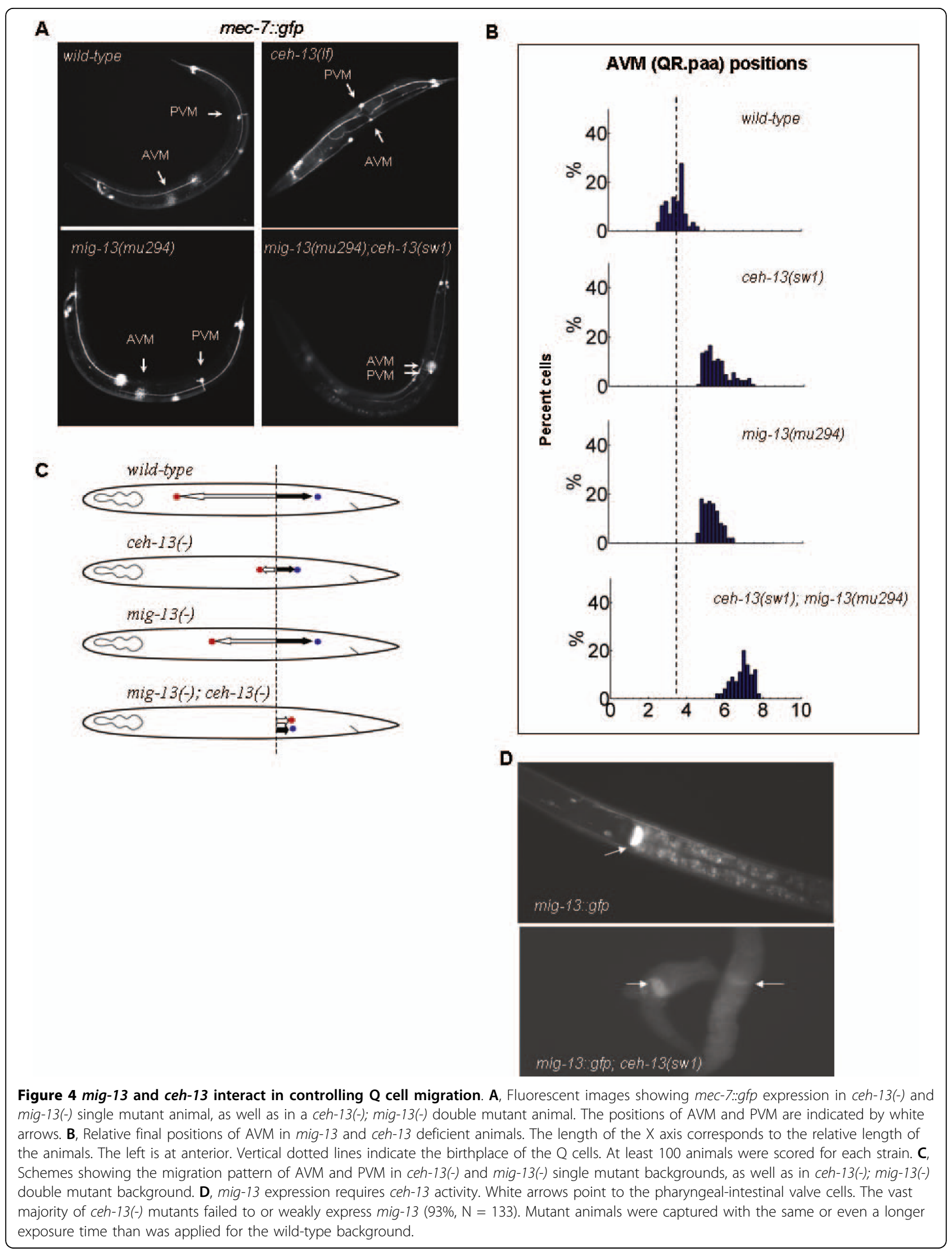


ceh-13 regulates the fusion process of Pn.p cells in both anterior and posterior body parts

The fusion of certain epidermal blast cells with the hypodermal syncytium hyp7 represents another example, in which the distinct and combined regulatory functions of lin-39 and mab-5 have been well characterized [11,12]. At the early L1 larval stage, the ventral epidermis is composed of 12 ectodermal precursor cells, termed $\mathrm{P}(1-12)$, which are located in a row along the ventral surface of the animal (Figure 5A) [27]. At the late L1 stage, the $P$ cells divide once, generating the Pn.a neuroblast and Pn.p epidermal daughters. Soon after their birth, some of the Pn.p cells fuse with hyp7, while the others remain unfused. The fusion pattern of Pn.p cells is established in a sex-specific manner, and regulated by lin-39 and mab5. In wild-type hermaphrodites, the central $\mathrm{P}(3-8) . \mathrm{p}$ cells
A

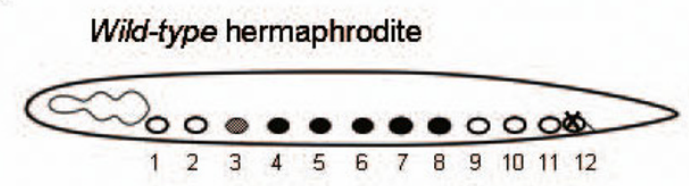
ceh-13(sw1)

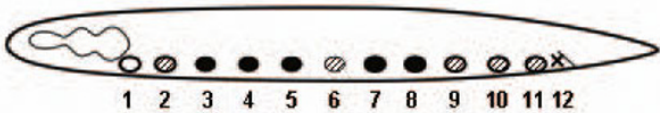

c
B
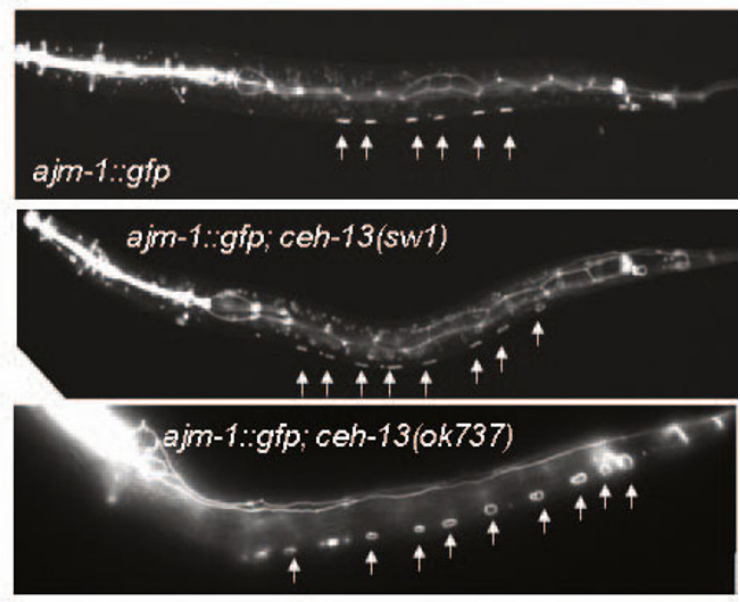

Pn.p cells

\begin{tabular}{lccccccccccc}
\cline { 2 - 7 } Genotype & 1 & 2 & 3 & 4 & 5 & 6 & 7 & 8 & 9 & 10 & 11 \\
\hline Wild-fype & 0 & 0 & 0 & 0 & 0 & 0 & 0 & 0 & 0 & 0 & 0
\end{tabular}

(\%) $0 \quad 0 \quad 63100100 \quad 100 \quad 100100 \quad 0 \quad 0 \quad 0$

\begin{tabular}{|c|c|c|c|c|c|c|c|c|c|c|}
\hline \multirow[t]{2}{*}{ ceh-13(swi) } & & 0 & $\theta$ & 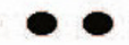 & 0 & & 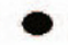 & $\varnothing$ & 0 & 0 \\
\hline & (\%) & 0 & 8100 & 100100 & 95 & 100 & 100 & 5 & 23 & 19 \\
\hline \multirow[t]{2}{*}{ ceh-13(ok737) } & & 0 & 0 & & 0 & & & 0 & 0 & \\
\hline & (\%) & 0 & 12100 & 100100 & 97 & 100 & 100 & 11 & 19 & \\
\hline mab-5(e1751gf) & & 0 & 0 & & - & 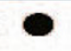 & & 0 & 0 & \\
\hline
\end{tabular}

Figure 5 ceh-13 promotes cell fusion in Pn.p lineages. A, Cell fusion pattern of the Pn.p cells in wild-type hermaphrodites at the L1 larval stage. Open circles represent Pn.p cells that fuse with the hypodermal syncytium hyp7, black circles represent Pn.p cells that remain unfused, shaded circle represents P3.p, which fuses with hyp7 only in nearly half of the animals. The Pn.p cells are indicated by numbering below the animal. B, An integrated ajm-1:.:gfp reporter outlines six unfused Pn.p cells, P(3-8).p, in a wild-type L2 stage larva (top), and, in addition to P(3-8).p, several other Pn.p cells that remain ectopically unfused in ceh-13(-)mutant L2 stage larvae (middle and bottom). The unfused Pn.p cells are indicated by white arrows. C, Effect of ceh-13 deficiency on the Pn.p cell fusion pattern. The fusion pattern in wild-type larvae, as well as in ceh$13(I f)$ and mab-5(gf) mutant larvae after the early fusion event at the L1/L2 larval stages (the numbers below the circles represents the percentage of the fusion events). 200 individual larvae were examined for each strain. 
are prevented from undergoing fusion by lin-39. The fusion pattern of these cells is also influenced by CEH-20 [25]. In hermaphrodites defective for this HOX cofactor, $\mathrm{P}(3-8) . \mathrm{p}$ and some additional anterior and posterior Pn.p cells remain unfused. The fusion defective phenotype of ceh-20 mutants is obvious even in lin-39 null mutant background [25]. This is particularly interesting since in lin-39 single mutant animals each Pn.p cell fuses with hyp7 [11,12].

To examine whether ceh-13 is also involved in this cell fusion event, we scored the number of unfused Pn.p cells in ceh-13 mutant animals. The lack of the fusion process was identified by an integrated ajm-1::gfp (apical junction molecule) reporter that is specific for a component of adherens junctions and thus outlines unfused cells [30]. We found that in addition to the central $\mathrm{P}(3-8)$.p cells, several anterior and posterior Pn.p cells remain unfused in ceh-13(sw1) hermaphrodites: $8 \%$ of P2.p, 23\% of P9.p and 19\% of P10.p $(\mathrm{N}=200)$ were unable to fuse with hyp7 in the mutant larvae examined (Figure 5B, C). ceh-13(ok737) mutants displayed similar defects in cell fusion (Figure 5B, C). Thus, the effect of ceh-13 on Pn.p fusion is not restricted to the anterior body part, but also extended to cells located in the posterior body region. The cell fusion defective phenotype of ceh-13 mutant larvae indicates that ceh-13 promotes the fusion of Pn.p cells with hyp7. We conclude that ceh-13 acts in an opposite way to LIN-39 to control this process. Interestingly, the fusion pattern of Pn.p cells in ceh-13 mutants highly resembles to that found in animals defective for ceh-20 [25]. These results suggest that ceh-13 is also involved in establishing the proper number of unfused Pn.p cells in hermaphrodites.

mab-5 does not affect Pn.p fusion in hermaphrodites $[11,12]$. However, the mab-5 gf mutation $e 1751$ was able to restore the fusion defect of Pn.p cells in ceh-13 mutant background (Figure 5C). In the ceh-13(sw1)mab5(e1751gf) double mutant animals that morphologically looked normal, the fusion pattern of the Pn.p cells appeared unaffected. Upon these results we suggest that ectopically or excessively expressed MAB-5 can substitute $\mathrm{CEH}-13$ in cell fusion control.

\section{ceh-13 influences vulval patterning}

In the C. elegans hermaphrodite, the vulval tissue through which the animal lays embryos - develops from a subset of six Pn.p epidermal cells [P(3-8).p] called vulval precursor cells (VPCs), which lie ventrally along the anterior-posterior axis [27,31]. Although each VPC has the potential to adopt an induced vulval fate, normally only the three central VPCs, P(5-7).p, undergo vulval induction. The non-induced VPCs, $\mathrm{P}(3,4,8) \mathrm{p}$, divide once and their daughters fuse with hyp7. Descendants of the induced VPCs form eventually the matured vulval structure. The fate of VPCs is determined by the combined effect of multiple genetic cascades, including the Ras, Wnt and Notch signaling pathways, signaling via TRA-1 (sexual transformer) that is similar to the Drosophila Cubitus interruptus and mammalian GLI(Glioma-associated) like proteins, and three redundant synMuv (for synthetic Multivulva) pathways grouped into classes A, B and C [31-34]. Under hyperinducing conditions, $\mathrm{P}(3,4,8)$. $\mathrm{p}$ can ectopically adopt induced vulval fates, which manifests in multiple vulval protrusions (Multivulva - Muv - phenotype). When vulval induction fails to occur, no VPC adopts vulval fate which renders the animal to exhibit a Vulvaless ( Vul) phenotype.

Both lin-39 and mab-5 affect vulval fate specification $[34,35]$. Furthermore, VPC induction in ceh-20 mutants can occur even in the complete absence of LIN-39 [25]. This knowledge prompted us to examine vulval morphology in ceh-13 mutant escapers. We found that these animals are Muv with a relatively low penetrance $(<1 \%$, $\mathrm{N}=950$ ). The affected adults had an extra vulval protrusion close to the normal vulva (Figure 6A). This phenotype is similar to that observed in lin-39 hypomorphic and ceh-20 lf mutants [26], suggesting that these factors, including ceh-13, have a similar role in VPC specification. To further assess this possibility, we monitored the effects of ceh-13 lf mutations on vulval induction in syn$M u v A B$ double mutant background. Single mutations in either of the SynMuv A or B pathway do not affect vulval development, whereas simultaneous inhibition of the two pathways renders the animals to be Muv [32]. Like lin-39 deficiency [36], inactivation of ceh-13 significantly suppressed vulval induction in synMuv $A B$ double mutant background (Figure 6B). Thus, ceh-13 endorses VPCs to adopt an induced vulval fate (note that this complex vulval morphology of ceh-13 If mutants - i.e., an extra vulval protrusion that is due to hypomorphic mutations and suppression of vulval induction in syn$M u v A B$ double mutant background by lf mutations - is also characteristic for lin-39 deficient animals [26]). It may exert this function by directly controlling some target "vulval" genes. Alternatively, the absence of ceh-13 activity may alter the expression domain of the middle Hox paralogs, especially that of lin-39, thereby influencing vulval patterning. Nevertheless, ceh-13 has obvious developmental roles in the mid-body region as well.

\section{The expression domain of ceh-13 overlaps with that of the other Hox paralogs}

Although ceh-13 is most similar to the anterior Hox orthologs, e.g., Drosophila labial and mammalian HoxB1, its functional domain is obvious all along the anteroposterior body axis (see results above). Indeed, a transcriptional fusion ceh-13::gfp reporter, pMF1, has been previously reported to be expressed in many 
A

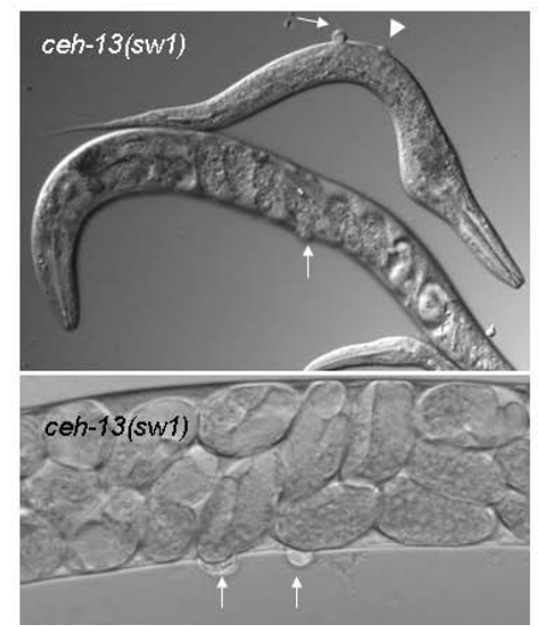

B

\begin{tabular}{|c|c|c|c|}
\hline \multirow{2}{*}{$\begin{array}{l}\text { Genotype } \\
\text { wild-type }\end{array}$} & \multicolumn{2}{|c|}{$\begin{array}{l}\text { Average number of } \\
\text { vulval protrusion }(\mathrm{N})\end{array}$} & \multirow[t]{2}{*}{$P$} \\
\hline & 1.0 & (many) & \\
\hline ceh-13(sw1) & 1.2 & (950) & \\
\hline ceh-13(ok737) & 1.1 & (185) & \\
\hline lin-8(n111); $\operatorname{lin-15B(n765ts)}$ & 3.89 & $(327)$ & $>0.0001$ \\
\hline $\operatorname{lin}-53(n 833) ;$ lin-15A(n765ts) & 3.67 & (165) & $>0.0001$ \\
\hline ceh-13(sw1); lin-8(n111); lin-15B(n765ts) & 2.29 & (344) & $>0.0001$ \\
\hline ceh-13(ok737); lin-8(n111); lin-15B(n765ts) & 3,04 & (70) & $>0.0001$ \\
\hline ceh-13(sw1); lin-53(n833); lin-15A(n765ts) & 2.81 & (162) & $>0.0001$ \\
\hline
\end{tabular}

Figure 6 ceh-13 affects vulval patterning. A, Vulval morphology in ceh-13(-) mutant hermaphrodites. White arrows indicate vulval protrusions (protruded vulval phenotype; Pvl), the arrowhead indicate an ectopic vulva (top). ceh-13(-) mutants exhibit variable vulval morphologies, including Pvl (8\%), Multivulva (Muv; 0.2\%) and Egg-laying defective (Egl; 14\%) phenotypes ( $\mathrm{N}=200)$. B, Mutational inactivation of ceh-13 decreases vulval induction in synMuv $A B$ double mutant animals. For each triple mutant: $P<0.001$; for statistics, see the Material and Methods. lin-8 and lin-15A are class A synMuv genes, while lin-53 and lin-15B are class $B$ synMuv genes. Single mutants defective in either of the synMuv $A$ or B pathways have normal vulval morphology (data not shown), whereas synMuv AB double mutant are Muv. Wild-type animals actually have no vulval protrusion (their normal vulval structure is considered as 1 protrusion in the figure).

different cell lineages even from the mid- and posterior body parts during development $[20,13,14]$. To understand better this unusual - i.e., non-colinear - activity domain of ceh-13, we examined its expression in relation to that of the other Hox paralogs (Figure 7). To this end, we first generated $g f p$-labeled translational fusion reporters for C. elegans Hox genes whose expression has not been determined by such a system (see the Methods). The reporters we generated contain at least 9-10 kb large upstream regulatory sequences and almost the entire coding regions, and were able to partially rescue the corresponding mutant phenotype (data not shown). In two-fold stage embryos, nob-1, php-3 and egl-5 each were expressed in the tail region, as expected or previously reported (Figure 7A). Therefore, these posterior Hox paralogs, similar to their Drosophila and mammalian orthologs, are active in the tail region exclusively. In good agreement with previous results $[8,12]$, LIN-39 accumulated in the mid- and posterior body region, whereas mab-5 was expressed in the posterior half of the body at the two-fold embryonic stage (Figure 7A). The expression of ceh-13, however, was evident in each of the main body domains, which is consistent with its functional properties. Based on these results, we suggest that ceh-13 is a unique C. elegans Hox gene in that it is expressed and functions in a non-colinear way. Its expression domain overlaps with those of the other Hox paralogs.

We also examined the expression of the C. elegans Hox genes at the L1 larval stage, and found a similar pattern that characterizes them during embryonic development: the posterior Hox paralogs were active in the tail, LIN-39 was expressed mainly in the mid-body region, the expression of $m a b-5$ was restricted to the domain located between the posterior and central body parts, whereas ceh-13 expression was apparent along the entire anteroposterior body axis (Figure 7B). ceh-13 expression, for example, was detectable in nearly each of the P blast cells (Figure 7B) and their daughters [13]. Different neuronal precursors as well as lateral hypodermal cells were also CEH-13::GFP-positive in each of the main body regions. Thus, the expression domain of ceh13 may have been extended from the anterior during evolution, presumably following the reciprocal translocation of ceh-13 and its closest Hox paralog, lin-39.

\section{Extra copies of lin-39 and mab-5 can suppress the pleiotropic Ceh-13 mutant phenotype}

ceh-13 single mutant animals that are able to develop into adulthood are small, exhibit a variable abnormal morphology and reduced fertility, and have a slow growth rate [14]. As the expression domain of ceh-13 overlaps with those of the other C. elegans Hox paralogs, one might expect functional redundancy between the anterior Hox gene and other Hox paralogs. Indeed, we found that a translational fusion LIN-39::GFP reporter, zhIs1, is able to rescue larval, but not embryonic, lethality of ceh-13 mutants (note that this transgene was able to rescue the Vul phenotype of lin-39 hypomorph mutants). Whereas less than $4 \%(13 / 437)$ of the ceh-13(sw1) mutant larvae 


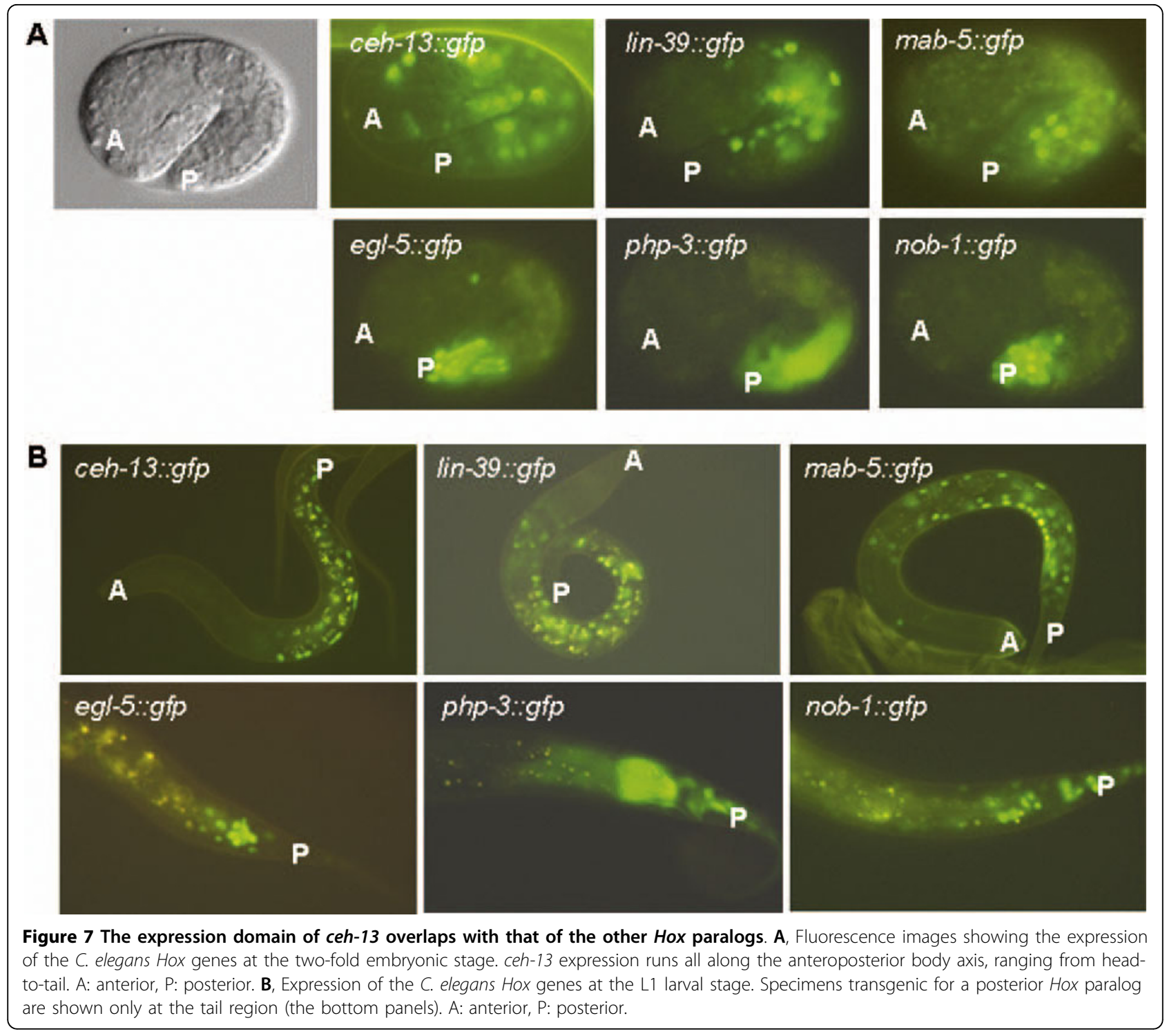

developed into fertile adults only, a large portion of ceh13 mutants bearing $z h I s 1$ were able to pass the larval stages (Figure 8A, B). In addition, the growth rate of ceh13 mutants was significantly faster with the reporter than without the reporter (data not shown).

We also used a mab-5 gf mutation to test functional redundancy between ceh-13 and this middle Hox paralog. We found that the majority of ceh-13(sw1)mab-5 (e1751gf) double mutants develop normally (Figure 8A, $B)$. The morphology and behavior of these animals appeared to be superficially wild-type. Consistently, the lifespan of ceh-13(sw1)mab-5(e1751gf) double mutant animals were also comparable with that of the wild type (Figure 8C). Thus, increased dosage of lin-39 and mab-5 may substitute ceh-13 function in certain developmental processes. This can explain why half of the mutants defective for ceh-13 are able to pass embryonic development, the existence of ceh-13 escapers, as well as the viability of lin-39mab-5 lf double mutants.

We also considered the possibility that ceh-13 is involved in regulating the expression of lin-39 or mab-5 in certain cell types. To address this issue we monitored mab-5 activity in ceh-13(sw1) mutant background, and found significant changes in the expression of mab-5, as compared with the wild-type background. For example, at the L1 larval stage mab-5 was ectopically expressed in the head, while its expression was strongly reduced in the tail region of ceh-13 mutants (Figure 8D). In contrast, lin-39 expression was hardly changed in ceh-13 mutants. We conclude that a complex - region- and stage-specific - regulatory relationship exists between ceh-13 and the middle Hox paralogs. 


\begin{tabular}{lcccc} 
A & N & $\begin{array}{c}\text { Dead } \\
\text { embryos }\end{array}$ & $\begin{array}{c}\text { Larval } \\
\text { arrest }\end{array}$ & Adults \\
\hline Genotype & 495 & $0 \%$ & $0 \%$ & $100 \%$ \\
Wild-type & 517 & $34 \%$ & $51 \%$ & $15 \%$ \\
ceh-13(sw1) & 300 & $26 \%$ & $56 \%$ & $18 \%$ \\
ceh-13(ok737) & 391 & $24 \%$ & $33 \%$ & $43 \%$ \\
ceh-13(sw1)mab-5(e1751gf) & 365 & $0 \%$ & $0 \%$ & $100 \%$ \\
mab-5(e1751gt) & & & &
\end{tabular}

\begin{tabular}{lrcrc}
\hline $\begin{array}{l}\text { B } \\
\begin{array}{l}\left(25^{\circ} \mathrm{C}\right) \\
\text { Genotype }\end{array}\end{array}$ & N & $\begin{array}{c}\text { Dead } \\
\text { embryos }\end{array}$ & $\begin{array}{r}\text { Larval } \\
\text { arrest }\end{array}$ & Adults \\
\hline Wild-type & 763 & $0 \%$ & $0 \%$ & $100 \%$ \\
ceh-13(sw1) & 647 & $38 \%$ & $58 \%$ & $4 \%$ \\
lin-39(+++) & 1088 & $15 \%$ & $0.09 \%$ & $84.9 \%$ \\
ceh-13(sw1); lin-39(+++) & 621 & ND & $19.5 \%$ & $20.4 \%$ \\
ceh-13(sw1)mab-5(e1751gf) & 562 & $33 \%$ & $30 \%$ & $37 \%$ \\
mab-5(e1751gf) & 488 & $1 \%$ & $0 \%$ & $99 \%$ \\
\end{tabular}
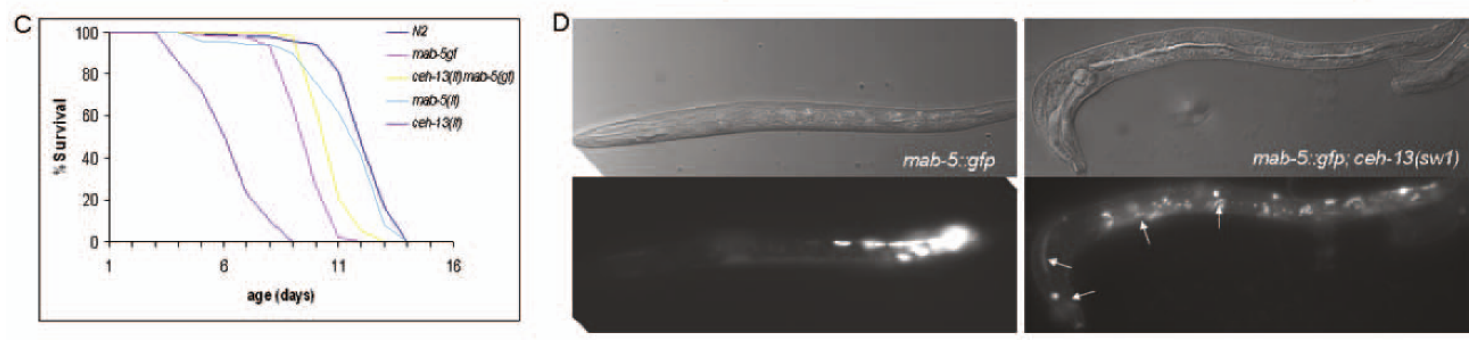

$\mathrm{E}$

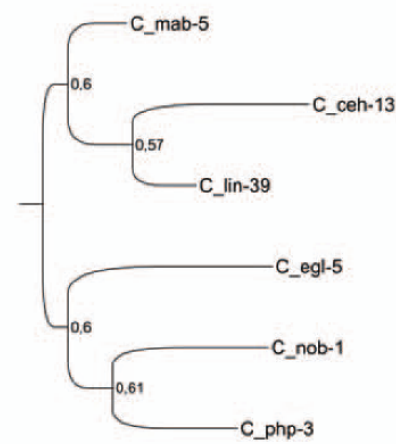

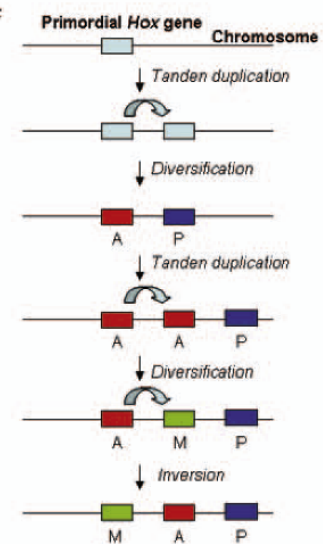

Figure 8 Increased activity of lin-39 and mab-5 can rescue some defects in ceh-13 mutant animals. A-B, A gain-of-function mutation in mab-5, e1751, suppresses lethality in ceh-13(-) mutants. Note that most of the double mutant animals look superficially normal. B, Extra copies of lin-39 suppress larval lethality in ceh-13(sw1) mutants. "+++" represents an integrated translational fusion LIN-39::GFP reporter that is able to rescue the Vul phenotype of lin-39 hypomorph mutants ( $5 \%, N=122)$. ND, not determined. $\mathbf{C}$, mab-5(e1751gf) mutation restores the lifespan of ceh-13(-) mutants to a nearly normal level. Note that the vast majority of ceh-13(-) single mutants die at early stages of development (Figure 8A, B), and the survivals (escapers) live only few days as adults (i.e., they are extremely short-lived). For double mutants, normal looking L4 stage larvae were selected and then scored for survival (yellow curve). Samples were assayed in triplicates. For ceh-13(sw1) single mutants, 70 mutant escapers were examined in each assay. For the other strains, 150-150 animals were scored in each assay. $\mathrm{p}<0.001$, when ceh-13(If)mab-5(gf) double mutants were calculated to ceh-13(If) single mutants by pair-wise comparisons, and $p>0.5$ when double mutants were compared to the wild type. The log-rank test was used for comparison. N2 indicates wild-type. D, mab-5::gfp expression in wild-type (left panel) vs. ceh-13(sw1) mutant (right panel) background at the L1 larval stage. Nomarski pictures on the top, corresponding fluorescent images at the bottom. White arrows indicate cells that are gfp-positive. E, Phylogenetic tree of homeodomain sequences of the C. elegans Hox genes. The tree was generated by the Bayesian phylogenetic method. Numbers at the nodes correspond to the probability - i.e., clade credibility - of each node (from 0 to 1 ). F, Model for the early evolution of the nematode Hox cluster. A ceh-13-like primordial Hox gene underwent a tandem gene duplication event in an early phase of animal evolution, leading to the ancestors of the primitive anterior and posterior Hox genes. A subsequent tandem duplication of the anterior ancestor resulted in the ancestor of the middle-group Hox genes. A: anterior, P: posterior, M: Middle.

Finally, we performed a Bayesian phylogenetic analysis of the C. elegans Hox homeodomain sequences. On the tree generated the middle paralogs stem from the anterior Hox branch, further supporting their close relation to ceh-13 (Figure 8E). In the light of these finding, the role of ceh-13 in controlling the migration of posterior $\mathrm{Q}$ cell descendants, fusion of posterior Pn.p cells, and vulval induction is now better understandable. Together, these data provide a functional support for the common evolutionarily origin of ceh-13 and the middle Hox paralogs (Figure 8F).

\section{Discussion}

Genes from the Hox gene clusters encode evolutionarily conserved homeodomain-containing transcription factors that play a pivotal role in axial patterning during 
animal development [1-5]. C. elegans is an attractive model system to study the unique and combined effects of Hox genes on developmental processes. However, our present knowledge on the roles of Hox genes in this organism is rather unbalanced as numerous studies have shed light into the mechanisms by which three Hox genes, lin-39, mab-5 and egl-5, control various developmental events, but there is almost no information on the function of the other Hox paralogs, ceh-13, nob-1 and $p h p-3$. In this study we describe novel roles for ceh13 in different developmental processes, and show that its functional domain obvious along the anteroposterior body axis, representing a break in colinearity.

\section{ceh-13 influences $Q$ cell migration}

Our data presented here indicate that ceh-13 is required for the proper positioning of Q neuroblasts (Figs. 2, 3, 4). Inactivation of ceh-13 led to a shortened migration of the AVM (QR.paa) and PVM (QL.paa) cells. AVM was positioned improperly in nearly each of the ceh-13 deficient animals examined. Mislocalization of PVM was also obvious in a significant portion of ceh-13 mutant larvae. This latter cell migration defect is particularly intriguing as it manifests in the posterior body domain. Although ceh-13 is considered as an anterior (labial-like) Hox gene [14], its defect disrupted the ability of a QL descendant to migrate toward the posterior. Furthermore, elimination of ceh-13 function also caused mispositioining of ALM sensory neurons in the majority of ceh-13 mutants.

Upon these findings, it was relevant to ask whether ceh-13 interacts with mig-13, which affects AVM migration and whose expression is controlled by mab-5 [29]. In ceh-13(-); mig-13(-) double mutant animals, the migration of AVM was more severely affected than in mig-13 single mutants. Thus, the two genes may act parallel to control cell migration in the anterior body domain. Alternatively, ceh-13 influences both mig-13 activity and another pathway to affect this process. mig13 expression was abolished upon ceh-13 deficiency, supporting the latter possibility. These data are consistent with previous findings reporting that ceh-20 is required for mig-13 activity to control cell migration in a lin-39-independent manner [25]. It is worth to note, however, that the regulatory region of mig-13 contains no canonical binding site for CEH-13. Thus, the effect of $\mathrm{CEH}-13$ on mig-13 expression may be indirect. Together, we conclude that ceh-13 affects cell migration in body parts covered by functional domains of the middle Hox paralogs.

\section{ceh-13 affects the fusion of Pn.p cells in various body domains}

The fusion of Pn.p cells with hyp7 at the early larval stages is known to be under the control of lin-39 and mab-5 [11,12]. According to our present data, ceh-13 is also involved in this process at least in hermaphrodites (Figure 5). Unexpectedly, ceh-13, unlike lin-39, appeared to promote Pn.p fusion, and this function was evident even in the posterior body part; besides the central $\mathrm{P}(3-$ 8).p cells, additional Pn.p cells remained unfused in animals defective for $\mathrm{CEH}-13$. These results indicate that ceh-13 opposes with lin-39 in certain biological processes. It is possible that the two Hox genes share common targets genes mediating cell fusion, but regulate them in an opposite way. Alternatively, lin-39 represses genes required for cell fusion, such as eff-1 [37], while ceh-13 promotes the expression of another set of genes that promote cell fusion. Nevertheless, ceh-13 and lin-39 may co-ordinately regulate cell fusion in certain Pn.p lineages. This can explain why the fusion pattern of Pn. p cells is quite different between lin-39 and ceh-20 mutant hermaphrodites [25]. Whereas in lin-39 lf mutant larvae almost all Pn.p cells fuse with hyp7, in ceh-20(mu290) mutants each VPC [P(3-8).p] and some of the most anterior and posterior Pn.p cells remain unfused [25].

\section{ceh-13 affects vulva patterning}

lin-39 is required during the early larval stages for the $\mathrm{P}$ (3-8).p cells to remain unfused, and, later at the L3 larval stage, to generate vulval cell divisions $[11,12]$. Considering cell fusion, ceh-13, similarly to ceh-20 [25], appears to contrast with lin-39: it promotes, rather than prevents, fusion in Pn.p cells (see data above). The higher number of unfused Pn.p cells in ceh-13 lf mutants may be the reason of why these animals exhibited, although with a low penetrance, ectopic vulval induction (Figure 6). In controlling vulval cell division, however, ceh-13 acts along with lin-39; inactivation of ceh-13 reduced the ability of VPCs to undergo vulval induction in synMuv $A B$ double mutant background (Figure 6). Consistently, ceh-13 is known to be expressed in the major hypodermal syncytium [13], in which synMuv genes antagonize Rasmediated vulval induction [38]. It is also possible that ceh-13 indirectly affects vulval induction via modulating the expression of other Hox factors involved in vulval cell fate specification. Together, vulval patterning is another example in which the anterior Hox paralog ceh-13 acts in the middle body domain.

\section{Lethality of ceh-13 mutants can be suppressed by increased dosage of middle Hox paralogs}

Double mutant animals defective for both lin-39 and $m a b-5$ are viable and fertile. In contrast, the majority of ceh-13(-) single mutants arrest development at the embryonic or early larval stages [14]. In this study we show that extra copies of lin-39 or a gf mutation of mab-5 are able to rescue lethality in ceh-13 deficient animals. In 
addition, other aspects of the pleiotropic Ceh-13 phenotype, including malformations at various body parts, slow growth rate, reduced lifespan and lowered fertility, could also be restored to normal levels by mab-5(e1751gf) mutation (Figure 8). These results also indicate a functional redundancy between ceh-13 and the middle-group Hox paralogs in various body domains. As ceh-13 has an unusually wide expression and activity domain, extending from the head to tail, it may cover and substitute several functions of lin-39 and mab-5, making the middle Hox paralogs to be dispensable for development. In contrary, lin-39 and mab-5 operate in the middle and posterior body domains, respectively. Their site of action overlaps only partially with that of ceh-13. As a result, ceh-13 has evolved as a predominantly essential Hox gene.

\section{Conclusions}

The nematode Hox genes are thought to have undergone rapid divergent evolution, probably due to the adaptation of the lineage-driven mode of development [7]. ceh-13 is the only anterior (labial-like) paralog encoded by the $C$. elegans genome. However, many of its characteristics make ceh-13 as a rather atypical anterior Hox gene. First, it is located between the two middle Hox paralogs, downstream of $l i n-39$ and upstream of $m a b-5$. This unusual genomic organization of ceh-13 resulted from an ancient inversion event, presumably soon after the emergence of lin-39 and ceh-13 from their common ancestor (Figure 8F). Second, the spatial expression domain of ceh-13 largely overlaps with that of the other Hox paralogs. Thus, its relative localization within the cluster and functional domain are not collinear with each other.

ceh-13 plays multiple roles in C. elegans development. It controls cell adhesion, tissue patterning in different parts of the body (i.e., morphogenesis), cell fusion, cell migration, growth rate and fertility [[14]; this study]. Some of these functions, such as cell adhesion and growth, might have characterized the primordial Hox gene that gave raise the ancestor Hox cluster through a series of tandem gene duplication and subsequent diversification events in an early phase of animal evolution. In this scenario, a ceh-13like primordial Hox gene underwent gene duplication, resulting in an upstream and a downstream daughter that became the prototype of the first anterior paralog and the first posterior paralog, respectively. After a sufficient degree of diversification, these genes conferred anterior (head) and posterior (tail) polarizations for the host organism. The anterior paralog then underwent duplication again: the resulting downstream descendant evolved into the first middle Hox paralog, increasing moprhological complexity in the mid-body domain. The fact that a ceh13-like ortholog presents in all nematode lineages examined so far supports this scenario [7]. The early inversion event that occurred between the ancestral anterior and middle Hox paralogs in the C. elegans lineage may conserved the broad expression domain and diverse developmental roles of the anterior descendant. Another possible, but much unlikely, interpretation for the unusual properties of ceh-13 is that its ancestor might have acquired various functions secondarily during evolution. The question, however, remains open why the other nematode $H o x$ paralogs have avoided such evolutionary innovations.

\section{Methods}

\section{Genetics and strains}

Standard methods were used for culturing and manipulating Caenorhabditis elegans strains. Strains were raised at $20^{\circ} \mathrm{C}$, unless indicated. Wild-type worms correspond to Bristol, strain N2 [39]. The following mutant and transgenic strains were used in this study:

muIs35 [MEC-7::GFP, lin-15(+)] V

zhIs1 [LIN-39::GFP, unc-119(+)]IV; unc-119(e2498)III

swIs1 [ceh-13::gfp, rol-6(su1006)]II

muIs16 [MAB-5::GFP, dpy-20(+)]?; dpy-20(e1282)IV

bxls12 [egl-5::gfp]

jcIs1 [ajm-1::gfp, rol-6(su1006), unc-29(+)]IV; unc-29 (e193)I

muIs62 [pmig-13::gfp; lin-15(+)]?

mig-13(mu225)X; lin-15(n765ts)X; muIs62 [pmig-13::

GFP, lin-15(+)]

ceh-13(sw1)III

ceh-13(ok737)III

mab-5(e1751gf)III

mab-5(e1751gf)III; ceh-13(sw1)III

mab-5(e1751gf)III; muIs35 [MEC-7::GFP; lin-15(+)] V

mab-5(e1751gf)III; ceh-13(sw1)III; muIs35 [MEC-7::

GFP; lin-15(+)] $V$

mig-13(mu294)X; muIs35 [MEC-7::GFP; lin-15(+)] V

ceh-13(sw1)III; mig-13(mu294)X; muIs35 [MEC-7::GFP;

lin-15(+)] V

ceh-13(sw1)III; muIs62 [pmig-13::gfp; lin-15(+)]

ceh-13(sw1)III; muIs35 [MEC-7::GFP; lin-15(+)] V

ceh-13(ok737)III; muIs35 [MEC-7::GFP; lin-15(+)] V

let-60 (n1064gf)IV

lin-8(n111)II; lin-15B(n765ts)X

lin-53(n833)I; lin-15B(n765ts)X

ceh-13(sw1)III; let-60 (n1064gf)IV

ceh-13(sw1)II; lin-8(n111)II; lin-15B(n765ts)X

ceh-13(ok737)III; lin-8(n111)II; lin-15B(n765ts)X

ceh-13(sw1)III; lin-53(n833)I; lin-15B(n765ts)X

\section{Characterization of the mutation ok737}

ok737 allele is a deletional derivative of ceh-13. It removes the end of the first exon and more than half of the first intron, resulting in a frame shift and the generation of a premature stop codon 26 base pairs after the downstream deletional breakpoint. Based on phenotypic characterization (see below), ok737 is likely to 
represent another null allele of ceh-13. Homozygous ceh-13(ok737) mutant animals exhibit a variable abnormal morphology phenotype accompanied with a highly penetrant embryonic or larval lethality: $96 \%(621 / 647)$ of ok737 mutants die at various stages of embryogenesis or at early larval stages. The arrested embryos and larvae display serious body malformations, especially at the anterior and central body parts. The ceh-13(ok737) escapers, which are able to develop into fertile adults, show less severe morphological defects than the arrested mutants, and have a decreased brood size (data not shown), a short body length and delayed developmental rate, as compared with the wild type. Homozygous ceh-13(ok737) mutants were isolated from the VC509 balanced strain of genotype ceh-13(ok737) III/hT2[bli-4 (e937) let-?(q782) qIs48] (I;III). For sequencing the mutant allele, genomic PCR was performed with the following forward and reverse primers: 5 '-tga gct cca ctg aat gtt atg g-3' and 5'-tat gac gaa ccg gtc ttt cc-3'.

\section{Assaying the positions of $Q$ descendants}

The position of AVM (QR.paa) and PVM (QL.paa) was determined by an integrated mec-7::gfp reporter [28]. Their location was scored as described preciously $[25,29]$. Statistical analysis of cell distribution was performed by the software MATLAB. The position of PVM was also screened by using an integrated tax-4::gfp fusion reporter [29].

\section{Assaying cell fusion in Pn.p lineages}

Fused vs. unfused fates of Pn.p cells were determined at the end of the L1 larval stage by using an integrated ajm-1::gfp reporter [30], which marks a component of adherent junction, thereby labeling the outline of unfused cells. Analysis of vulval patterning was performed by differential interference contrast (DIC) microscopy. Images were collected from an Olympus BX-51 microscope equipped with an F-WU II camera. Statistical analysis of vulva phenotypes was performed by unpaired $t$-test, using the software SPSS 14.0.

\section{Construction of $g f p$ reporters}

A $15 \mathrm{~kb}$ large genomic fragment consisting of $10 \mathrm{~kb}$ upstream regulatory element and almost the entire nob-1 coding region except for the last codon, was PCR amplified by using the following primers. Forward primer: 5'aac tga gaa cca atg cat tgg ctc cta tca cgg ggt tct gg-3', reverse primer: 5'-cgg gat ccc ggt tga tca atc gct cga tgc-3'. The resulting fragment was digested with PstI and BamHI, and cloned into the expression vector pPD95.75. Transgenic lines were produced by microinjection, using the co-transformation marker rol-6(su1006). Extrachromosomal transgenes then was integrated by UV radiation and subsequent isogenization by crossing back with the wild type 8 times. For amplifying the $p h p-3$ coding regions, genomic PCR was performed by the following forward and reverse primers: 5 '-tgt ttc tca aaa acg gat gg-3' and 5 '-cgg gat ccc gcg tag gca gtt gtg cag ctc ttg tc-3'. The PCR fragment was digested with MluI and BamHI, and cloned into the nob-1::gfp-containing vector (Note that nob-1 and $p h p-3$ are adjacent genes and use the same promoter region for regulating expression). Transgenic worms containing the extrachromosomal array were generated as described above.

\section{Lifespan assays}

Lifespan assays were carried out at $25^{\circ} \mathrm{C}$. For synchronization, 20-30 gravid well-fed adults $(\mathrm{P})$ were transferred to a new agar plate containing nematode growth medium (NGM) seeded with E. coli OP50 to lay eggs for 4-5 hours, and then removed. F1 young (not gravid) adults were transferred to NGM plates supplemented with $300 \mathrm{mg} / \mathrm{ml}$ FUDR (5-fluoro-2'-deoxyuridine) for 1 day $(t=0)$. This treatment inhibited the germ-line to produce germ cells. Sterile F1 adults were then transferred to the final assay plates and scored. SPSS 14 software was used to calculate mean lifespan and perform statistical analysis. p values for comparing Kaplan-Meyer survival curves between two groups were determined using log-rank (Mantel-Cox) tests.

\section{Phylogenetic analysis}

For clustering the $H O X$ genes from C. elegans (AC numbers: ceh-13: NM_066254; egl-5: L19247; lin-39: L19248; mab-5: AF277990; nob-1: AF172090; php-3: AF172092), we applied the Bayesian phylogenetic method and used 180 nucleotide long conserved homedomain sequences. For calculating the tree, MrBayes v3.1.2 software was used. The codon likelihood model was applied with one substitution type and with invariable proportion of sites. Te rate for the remaining sites were drawn from an estimated gamma distribution. The number of generations was set 1.000 .000 and two independent runs were done. Values on the tree correspond to the posterior probability - i.e. clade credibility of each node [from 0 (minimum) to 1 (maximum)].

\section{Acknowledgements}

This work was supported by grants from the Hungarian Scientific Research Funds (OTKA PD75477) to K.T-V., the National Office for Research and Technology (TECH_08_A1/2-2008-0106) and the Ministry of Health (ETT 142/2009) to T.V., and SNF Grants (3100-068240 and 31003A-109938) to F.M. T.V. and K.T-V. are grantees of the János Bolyai scholarship. The authors declare that they have no conflicts of interest that relate to the publication of this paper.

\section{Author details}

${ }^{1}$ Department of Genetics, Eötvös Loránd University, Budapest, H-1117, Hungary. ${ }^{2}$ Department of Biology, University of Fribourg, Fribourg, $\mathrm{CH}-1700$, Switzerland. 


\section{Authors' contributions}

BT made the majority of the experiments, wrote the manuscript, performed statistical analysis. T. $\vee$ designed experiments, performed the expression analysis of the Hox genes, wrote the manuscript. A R performed vulval analysis in ceh-13 and synMuv mutant animals. EA performed the phylogenetic analysis of the nematode Hox genes. FM designed experiments, wrote the manuscript. KTV supervised the work, designed experiments, performed cloning experiments, wrote the manuscript. All authors read and approved the final manuscript.

Received: 12 April 2010 Accepted: 28 July 2010 Published: 28 July 2010

\section{References}

1. McGinnis W, Krumlauf R: Homeobox genes and axial patterning. Cell 1992, 2:283-302.

2. Duboule D, Morata G: Colinearity and functional hierarchy among genes of the homeotic complexes. Trends Genet 1994, 10:358-364.

3. Kenyon CJ, Austin J, Costa M, Cowing DW, Harris JM, Honigberg L, Hunter CP, Maloof JN, Muller-Immergluck MM, Salser SJ, Waring DA, Wang BB, Wrischnik LA: The dance of the Hox genes: Patterning the anteroposterior body axis of Caenorhabditis elegans. Cold Spring Harbor Symp Quant Biol 1997, 62:293-305.

4. Hueber SD, Lohmann I: Shaping segments: Hox gene function in the genomic age. Bioessays 2008, 30:965-979.

5. Gehring WJ, Kloter U, Suga H: Evolution of the Hox gene complex from an evolutionary ground state. Curr Top Dev Biol 2009, 88:35-61.

6. Van Auken K, Weaver DC, Edgar LG, Wood WB: Caenorhabditis elegans embryonic axial patterning requires two recently discovered posteriorgroup Hox genes. Proc Natl Acad Sci USA 2000, 97:4499-4503.

7. Aboobaker AA, Blaxter ML: Hox Gene Loss during Dynamic Evolution of the Nematode Cluster. Curr Biol 2003, 13:37-40.

8. Kenyon C: A gene involved in the development of the posterior body region of C. elegans. Cell 1986, 46:477-487.

9. Schaller D, Wittmann C, Spicher A, Müller F, Tobler H: Cloning and analysis of three new homeobox genes from the nematode Caenorhabditis elegans. Nucleic Acids Res 1990, 18:2033-2036.

10. Bürglin TR, Ruvkun G: The Caenorhabditis elegans homeobox gene cluster. Curr Opin Genet Dev 1993, 3:615-620.

11. Clark SG, Chisholm AD, Horvitz HR: Control of cell fates in the central body region of C. elegans by the homeobox gene lin-39. Cell 1993, 74:43-55.

12. Wang BB, Muller-Immergluck MM, Austin J, Robinson NT, Chisholm A, Kenyon C: A homeotic gene cluster patterns the anteroposterior body axis of C. elegans. Cell 1993, 74:29-42.

13. Wittmann C, Bossinger O, Goldstein B, Fleischmann M, Kohler R, Brunschwig K, Tobler H, Müller F: The expression of the C. elegans labiallike Hox gene ceh-13 during early embryogenesis relies on cell fate and on anteroposterior cell polarity. Development 1997, 124:4193-4200.

14. Brunschwig K, Wittmann C, Schnabel R, Burglin TR, Tobler H, Müller F: Anterior organization of the Caenorhabditis elegans embryo by the labial-like Hox gene ceh-13. Development 1999, 126:1537-1546.

15. Wrischnik LA, Kenyon CJ: The role of lin-22, a hairy/enhancer of split homolog, in patterning the peripheral nervous system of $\mathrm{C}$. elegans. Development 1997, 124:2875-2888.

16. Salser SJ, Kenyon C: Activation of a C. elegans Antennapedia homologue in migrating cells controls their direction of migration. Nature 1992, 355:255-258

17. Maloof JN, Kenyon C: The Hox gene lin-39 is required during C. elegans vulval induction to select the outcome of Ras signaling. Development 1998, 125:181-190.

18. Liu H, Strauss TJ, Potts MB, Cameron S: Direct regulation of egl-1 and of programmed cell death by the Hox protein MAB- 5 and by CEH-20, a $C$. elegans homolog of Pbx1. Development 2006, 133:641-650.

19. Streit A, Kohler R, Marty T, Belfiore M, Takacs-Vellai K, Vigano MA, Schnabel R, Affolter M, Müller F: Conserved regulation of the Caenorhabditis elegans labial/Hox1 gene ceh-13. Dev Biol 2002, 2:96-108.

20. Stoyanov CN, Fleischmann M, Suzuki Y, Tapparel N, Gautron F, Streit A, Wood WB, Müller F: Expression of the C. elegans labial orthologue ceh-13 during male tail morphogenesis. Dev Biol 2003, 259:137-149.
21. Mann RS, Chan SK: Extra specificity from extradenticle: the partnership between HOX and PBX/EXD homeodomain proteins. Trends Genet 1996, 12:258-262.

22. Mann RS, Lelli KM, Joshi R: Hox specificity unique roles for cofactors and collaborators. Curr Top Dev Biol 2009, 88:63-101.

23. Liu J, Fire A: Overlapping roles of two Hox genes and the exd ortholog ceh-20 in diversification of the C. elegans postembryonic mesoderm. Development 2000, 23:5179-5190.

24. Van Auken $K$, Weaver $D$, Robertson $B$, Sundaram $M$, Saldi $T$, Edgar $L$, Elling U, Lee M, Boese Q, Wood WB: Roles of the Homothorax/Meis/Prep homolog UNC-62 and the Exd/Pbx homologs CEH-20 and CEH-40 in C. elegans embryogenesis. Development 2002, 22:5255-5268.

25. Yang L, Sym M, Kenyon C: The roles of two C. elegans HOX co-factor orthologs in cell migration and vulva development. Development 2005, 132:1413-1428.

26. Takács-Vellai K, Vellai T, Chen EB, Zhang Y, Guerry F, Stern MJ, Müller F: Transcriptional control of Notch signaling by a HOX and a PBX/EXD protein during vulval development in C. elegans. Dev Biol 2007, 302:661-669.

27. Sulston JE, Horvitz HR: Post-embryonic cell lineages of the nematode, Caenorhabditis elegans. Dev Biol 1977, 56:110-156.

28. Hamelin M, Scott IM, Way JC, Culotti JG: The mec-7 beta-tubulin gene of Caenorhabditis elegans is expressed primarily in the touch receptor neurons. EMBO J 2002, 11:2885-2893.

29. Sym M, Robinson N, Kenyon C: MIG-13 positions migrating cells along the anteroposterior body axis of C. elegans. Cell 1999, 98:25-36.

30. Mohler WA, Simske JS, Williams-Masson EM, Hardin JD, White JG: Dynamics and ultrastructure of developmental cell fusions in the Caenorhabditis elegans hypodermis. Curr Biol 1998, 8:1087-1090.

31. Sternberg PW: Vulval development. WormBook The C. elegans Research Community, WormBook 2005.

32. Harrison MM, Ceol CJ, Lu X, Horvitz RH: Some C. elegans class B synthetic multivulva proteins encode a conserved LIN-35 Rb-containing complex distinct from a NuRD-like complex. Proc Natl Acad Sci USA 2006, 103:16782-16787.

33. Szabó E, Hargitai B, Regős Á, Tihanyi B, Barna J, Borsos É, Takács-Vellai K Vellai T: TRA-1/GLI controls the expression of the Hox gene lin-39 during C. elegans vulval development. Dev Biol 2009, 330:339-348.

34. Hargitai B, Kutnyánszky V, Blauwkamp TA, Steták A, Csankovszki G, TakácsVellai $K$, Vellai T: $x o l-1$, the master sex switch gene in C. elegans, is a transcriptional target of the terminal sex-determining factor TRA-1/GLI. Development 2009, 136:3881-3887.

35. Clandinin TR, Katz WS, Sternberg PW: Caenorhabditis elegans HOM-C genes regulate the response of vulval precursor cell to inductive signal. Dev Biol 1997, 182:150-161.

36. Chen Z, Han M: C. elegans Rb, NuRD, and Ras regulate lin-39-mediated cell fusion during vulval fate specification. Curr Biol 2001, 11:1874-1879.

37. Shemer G, Podbilewicz B: LIN-39/Hox triggers cell division and represses EFF-1/fusogen-dependent vulval cell fusion. Genes Dev 2002, 16:3136-3141.

38. Myers TR, Greenwald I: lin-35 Rb acts in the major hypodermis to oppose ras-mediated vulval induction in C. elegans. Dev Cell 2005, 8:117-123.

39. Brenner S: The genetics of Caenorhabditis elegans. Genetics 1974, 77:71-94.

\section{doi:10.1186/1471-213X-10-78}

Cite this article as: Tihanyi et al: The C. elegans Hox gene ceh-13 regulates cell migration and fusion in a non-colinear way. Implications for the early evolution of Hox clusters. BMC Developmental Biology 2010 10:78. 